\title{
THE USE OF POLARIZATION EFFECTS IN SEMICONDUCTOR OPTICAL AMPLIFIERS TO PERFORM ALL-OPTICAL SIGNAL PROCESSING
}

\section{EL USO DE EFECTOS DE POLARIZACIÓN EN AMPLIFICADORES ÓPTICOS SEMICONDUCTORES PARA REALIZAR EL PROCESAMIENTO DE SEÑALES EN EL DOMINIO ÓPTICO}

\author{
Brendan F. Kennedy ${ }^{1} \quad$ Frederic Surre $^{2,3} \quad$ Severine Philippe $^{3} \quad$ Louise Bradley $^{3}$ \\ Recibido 9 de agosto de 2007, aceptado 10 de octubre de 2007 \\ Received: August 9, $2007 \quad$ Accepted: October 10, 2007
}

Pascal Landais ${ }^{2}$

\begin{abstract}
RESUMEN
En los servicios de telecomunicaciones, la necesidad de hacer uso intensivo de las aplicaciones ha presionado por un constante incremento del ancho de banda. Particularmente una de las tecnologías más promisorias, que ha permitido estos incrementos de ancho de banda, son los elementos ópticos, de tal manera que en las redes de comunicación los elementos electrónicos sean sustituidos por dichos elementos ópticos. En esta publicación se discute una técnica basada en la rotación no lineal del estado de polarización de una señal óptica conectada al amplificador semiconductor óptico. Se presenta un experimento, basado en este efecto, para realizar la conversión a 2,5 Gbits/s. La característica del conversor de longitud onda es probada en propagación directa e inversa. Se ha encontrado que conversión de la longitud de onda es independiente de longitud de onda (la conversión no depende del valor de la longitud de onda) en la modalidad de propagación directa no invertida.
\end{abstract}

Palabras clave: Amplificador semiconductor óptico, conversión de la longitud de onda, rotación no lineal del estado de polarización.

\section{ABSTRACT}

As the need for higher and higher bandwidths in telecommunication systems continues, it is widely predicted that at some point in the future optical processing will need to be performed all-optically. Several techniques have been proposed to perform such all-optical signal processing. In this paper a technique based on the nonlinear rotation in the state of polarization of an optical signal injected into a bulk semiconductor optical amplifier is discussed. An experiment to perform wavelength conversion at $2.5 \mathrm{Gbit} / \mathrm{s}$ based on this effect is presented. The performance of the wavelength converter is tested in both co-and counter-propagation, and wavelength independent wavelength conversion is found in the non-inverted co-propagation setup.

Keywords: Nonlinear polarization rotation, semiconductor optical amplifier, wavelength conversion.

\section{INTRODUCTION}

The recent explosive growth in internet traffic continues to increase with no sign of a levelling off in internet usage. In Chile, for example, the user penetration is the highest in South America with over $42 \%$ of the population using the internet. This represents a growth rate of over $200 \%$ in the last 5 years, and is a prime example of the exponential growth in internet usage in the world today. Densely populated countries with rapidly developing economies, for example India and China, will fuel the continued development of the internet. In the same five year period between 2000 and 2005, India's user growth was $684 \%$ resulting in a user penetration of only $3 \%$ whilst China's user growth was $358 \%$ resulting in a user penetration of only $7.9 \%$ [1]. As well as this, there is a continued rapid growth in the demand for new bandwidth hungry multimedia services. These facts indicate a future demand for faster switching capabilities than existing network infrastructure can provide.

\footnotetext{
1 Departamento de Ingeniería Eléctrica. Universidad de Santiago de Chile. Santiago, Chile. E-mail: bkennedy@lauca.usach.cl

2 Department of Electronic Engineering. Dublin City University. Ireland.

3 Physics Department. Trinity College Dublin. Ireland
} 
In long-haul telecommunications systems data propagates along optical fibre in optical format. The current systems, however, do not operate solely in the optical domain, as switching needs to be performed in the electronic domain. This involves conversion of the optical data to electronic data in order for the switching to take place and then conversion back to optical data after the switching operation has been performed. Commercial systems are currently restricted by the maximum speed at which the electronics can operate, which is $10 \mathrm{~Gb} / \mathrm{s}(\mathrm{OC}-192)$ [2], with the latest improved electronics operating at 40Gb/s (OC-768) [3]. As the overall data transmission obtainable in the optical domain far exceeds this maximum, electronic bottlenecks may occur in future systems at the multiplexer, electronic to optical converter, optical to electronic converter and at the demultiplexer. This would have the effect of limiting the maximum transmission rate of the system. In order to avoid these bottlenecks, at some point it will likely be necessary for high-speed telecommunication networks to operate solely in the optical domain. In order for this to be realized, techniques to perform this all-optical switching need to be developed.

The Semiconductor Optical Amplifier (SOA) has received extensive study as a potential device to implement all-optical signal processing. The SOA is a single-pass device, which is similar to a semiconductor laser operating below threshold. The device is kept below threshold by applying Anti-Reflection (AR) coatings and by tilting the waveguide of the device. Several techniques can be implemented using the SOA, namely Cross-Gain Modulation (XGM), Cross-Phase Modulation (XPM) and Four-Wave Mixing (FWM) [4]. These techniques take advantage of a modulation of either the gain or the phase of the device in order to perform all-optical signal processing, however, each one is limited by the polarization sensitivity in the SOA. The technique presented in this paper aims to take advantage of this inherent polarization sensitivity using the Nonlinear Polarization Rotation (NPR) effect in the device to perform Cross-Polarization Modulation (XPolM) [5, 6]. This effect occurs when a signal with a known state of polarization is injected into an SOA. Due to several effects in the device the state of polarization at the output of the device becomes rotated. This rotation is a nonlinear effect and may be used to perform all-optical signal processing such as wavelength conversion, where the data on a high intensity pump signal is transferred to a low intensity $\mathrm{CW}$ signal at a different wavelength. Experiments are presented in this paper in which wavelength conversion is performed in both co- and counter-propagation in order to determine which is the most efficient for the experimental setup under test. From these experiments it is found that for non-inverted conversion in the co-propagation setup it is possible to achieve wavelength conversion that is independent of the wavelength of the probe signal. This paper is organized as follows: Firstly, the causes of the NPR effect are introduced. After this an experiment is presented which demonstrates XPolM based on NPR in a bulk SOA at a data rate of $2.5 \mathrm{Gbit} / \mathrm{s}$.

\section{THE CAUSES OF NPR IN A BULK SOA DEVICE}

NPR in the SOA is caused by several effects. One of the principle causes is due to waveguide asymmetry in the device. If the device is not perfectly square the result is that the confinement factor, $\Gamma$, is different for the Transverse Electric (TE) and Transverse Magnetic (TM) Modes. This results in a polarization dependence of the device gain due to the equation (1):

$$
G=\left(\Gamma g_{m}-\alpha\right) L
$$

where $\Gamma$ represents the confinement factor, $g_{m}$ represents the material gain, $\alpha$ represents the optical losses and $\mathrm{L}$ is the length of the device.

Another significant type of polarization dependence present in an SOA with an asymmetric waveguide is due to the birefringence introduced to the device. This asymmetry causes two propagation constants to exist corresponding to the orthogonal TE and TM modes. The effective refractive indices typically differ by $2 \times 10^{-2}$ [7]. The difference in refractive index causes the TE and TM modes to propagate through the device at different speeds causing a phase difference to be introduced between the signals. An index difference as small as $2 \times 10^{-4}$ has been reported as being sufficiently high to induce a phase shift between the TE and TM modes of $90^{\circ}$ [6]. Many of the initial publications based on NPR justified the effect as being solely due to the asymmetric waveguide $[5,6]$. These papers also report an increase in the NPR due to birefringence as a function of injected power in the SOA. This modulation of the refractive index through modulation of the gain of the device is defined by the Self-phase Modulation (SPM) [8] and is quantified using the Linewidth Enhancement Factor (LEF) [9].

Because of the fact that the overall device gain is generally larger for the case of TE polarization, due to the difficulties in designing a completely square waveguide [10], a technique generally used is to introduce tensile strain to enhance the Light-Hole ( $\mathrm{LH}$ ) transitions, and therefore 
the TM transitions, in order to minimize the variation in gain between TE and TM axes. In this way the TM gain is increased to a level where it compensates for the higher TE gain. This balancing of the gains can be understood by considering the following expression [11]:

$$
\Delta G_{T E-T M}(d B)=\left\{1-\left(\frac{\Gamma_{T M}}{\Gamma_{T E}}\right)\left(\frac{g_{T M}}{g_{T E}}\right)\right\} G_{T E}(d B)
$$

Where $\mathrm{G}_{\mathrm{TE} / \mathrm{TM}}$ represents the modal gain, $\Gamma_{\mathrm{TE} / \mathrm{TM}}$ represents the confinement factor and $\mathrm{g}_{\mathrm{TE} / \mathrm{TM}}$ represents the material gain. From this expression it is clear that increasing $g_{T M}$ over $\mathrm{g}_{\mathrm{TE}}$ can compensate for the larger confinement along the $\mathrm{TE}$ axis. This is effective and although complete polarization insensitivity has never been achieved, it is an effective technique in compensating for the higher TE gain. However, it should be noted that the measurements performed to test this technique, from the literature, only consider the static steady-state gain and not the dynamic gain processes, which are governed by the material gain. This is of vital importance, as it is the response of the gain to data signals that is of interest for optical signal processing.

These are considered to be the strongest effects behind the NPR effect. One groups results also point to the modification of the eigenmodes of the device as being a cause of NPR [6]. This modification of the waveguide is brought about by a high intensity signal injected into the device causing a nonlinear birefringence effect. It was noticed that the TE and TM eigenmodes of the device at low injection powers were no longer the eigenmodes when the power was increased to high intensity values. Finally, in early SOA devices the polarization dependence of the AR coatings had a large effect, with a polarization dependence of over $10 \mathrm{~dB}$ being measured. Since this time, multi-layer coatings have been developed to reduce the polarization dependency due to AR coatings to below $10^{-4}$ [12].

\section{EXPERIMENTAL DEMONSTRATION OF XPOLM AT 2.5 GBIT/S}

After establishing the main causes of the NPR effect in a bulk SOA device, an experiment to perform wavelength conversion based on XPolM is now presented. Wavelength conversion is performed using XPolM at a data rate of $2.5 \mathrm{Gbit} / \mathrm{s}$ and a Pseudo Random Bit Sequence (PBRS) of $2^{7}-1$. This data rate is limited by the speed of the pseudo-random data generator and the error detector. The co-propagation setup is shown in figure 1. Two optical signals are simultaneously injected into the SOA. The SOA under test is an Avanex A1901, a tensile strained bulk amplifier structure with a gain spectral bandwidth of $75 \mathrm{~nm}$, centred at $1535 \mathrm{~nm}$. It is biased at $200 \mathrm{~mA}$ and temperature controlled at $23^{\circ}$. Both optical signals are generated using external cavity tunable lasers. The wavelength of the pump signal is $1538 \mathrm{~nm}$ and the wavelength of the probe signal is varied between $1535 \mathrm{~nm}$ and $1570 \mathrm{~nm}$. The span was limited to $35 \mathrm{~nm}$ due to the spectral bandwidth of the EDFAs, which operate in the C-band. The pump signal is modulated at $2.5 \mathrm{~Gb} / \mathrm{s}$ and then amplified through EDFA 2 to an average power of approximately $7 \mathrm{dBm}$ at the input of the SOA, which saturates the gain of the device. A BandPass Filter (BPF 2) is used to remove noise introduced by the EDFA. The probe signal is injected with a power of $-3.6 \mathrm{dBm}$. The signals are launched into the device in copropagation using a 50:50 coupler. The states of polarization, of both the pump and probe signals, are controlled using Polarization Controllers (PCs), which are adjusted in order to optimize the wavelength conversion. At the output of the SOA the combination of PC 3 and the PBS act to control the transmission of the amplified signal, based on the polarization of the signal. It is the combination of these two components that distinguishes this setup from that of XGM. The PBS used has an extinction ratio of $20 \mathrm{~dB}$ and an insertion loss of $0.4 \mathrm{~dB}$. The wavelength conversion due to XPolM is based on the fact that the polarization rotation is a nonlinear process. Both inverted and non-inverted XPolM can be performed using this setup by adjusting PC3 to maximize or minimize the transmission of the probe signal, in the absence of the pump signal. In the case of inverted XPolM, for example, the probe transmission is maximized before injection of the pump. It is necessary to include BPF 1 after the PBS in order to select the probe signal wavelength. This BPF has a $3 \mathrm{~dB}$ bandwidth of 1 $\mathrm{nm}$ and a wavelength tuning range of $40 \mathrm{~nm}$. EDFA 1 is used to amplify the wavelength converted signal and an attenuator is used to vary the received power falling on the photodetector. The signal can then be observed on an oscilloscope and Bit Error Rate Tester (BERT).

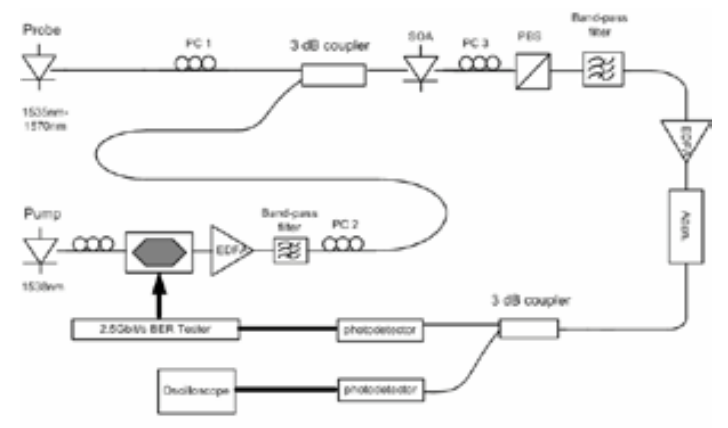

Figure 1. Co-propagation XPolM experimental setup. Bold lines represent electrical connections. 
The counter-propagation setup is shown in figure 2 . The operating conditions of the device and the pump and probe powers are the same as for the co-propagation setup. The pump and probe are injected into the device in opposite directions. A circulator with 35 $\mathrm{dB}$ isolation is used to inject the pump signal and to collect the converted signal. An isolator is used at the probe end of the device to ensure that the pump signal is not injected into the probe laser. Using this setup it is possible to convert data to the same wavelength. Because the signals are injected at opposite ends of the device it is not necessary to select the correct wavelength using BPF 1, but this filter is included to remove any Amplified Spontaneous Emission (ASE) that may have been transmitted through the PBS. Other than the opposite propagation direction of the data signal, the co- and counter-propagation setups operate in the same manner. In order to make a comparison between both techniques it is important to ensure that the pump and probe signals are injected into the device with the same level of intensity. It should be noted that both co- and counter-propagation setups can be easily changed to a XGM setup by removing PC 3 and the PBS, respectively. The wavelength dependence of XPolM is determined in both experimental setups for inverted and non-inverted conversion. The probe signal is varied from $1535 \mathrm{~nm}$ to $1570 \mathrm{~nm}$, whilst the pump signal is maintained at 1538 $\mathrm{nm}$. The pump wavelength is fixed as its main function is to deplete the carrier density in order that the device operates in the nonlinear regime. The probe wavelength is varied in order to measure the range of operation of the wavelength conversion scheme.

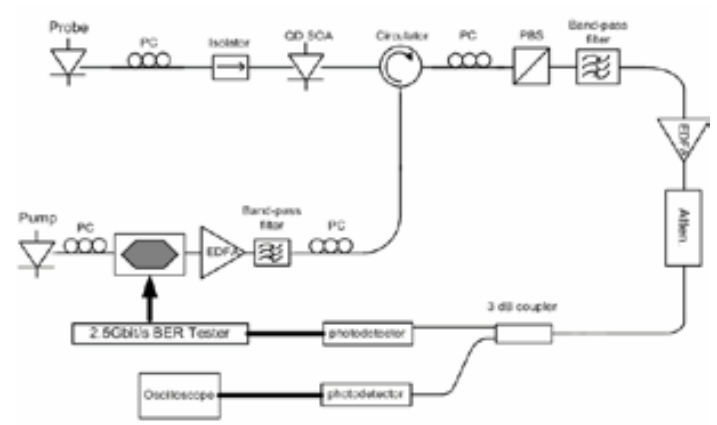

Figure 2. Counter-propagation XPolM experimental setup. Bold lines represent electrical connections.

A parameter known as the Q-factor is used to assess the performance of the wavelength conversion in this paper. The Q-factor is defined as:

$$
Q=\frac{I_{1}-I_{0}}{\sigma_{1}+\sigma_{0}}
$$

where $I_{1}$ and $I_{0}$ represent the mean signal level at ' 1 ' and ' 0 ', and and represent the standard deviation of the noise on the ' 1 ' and ' 0 ' level. It is measured using an oscilloscope. It should also be stated that the Q-factor is not directly related to the BER because of the non-Gaussian distribution of the intensity after the wavelength conversion. However, an approximation of the BER can be obtained from the Q-factor. The BER is measured in this experiment using a Bit Error Rate Tester (BERT) as illustrated in figure 1 and figure 2.

\section{ANALYSIS OF WAVELENGTH CONVERSION}

The Bit Error Rate (BER) as a function of received power for inverted and non-inverted XPolM, as well as XGM, is shown in figure 3 for both co- and counterpropagation schemes. The back-to-back data is also shown for comparison. In the co-propagation setup a penalty of approximately $1 \mathrm{~dB}$ is introduced for both inverted XPolM and XGM as can be seen in figure 3(a). However, a larger penalty of approximately $5.5 \mathrm{~dB}$ is introduced using the non-inverted XPolM in the co-propagation setup. This poor performance is a consequence of XGM, which occurs simultaneously with XPolM. XGM leads to inverted conversion and therefore opposes the effect of non-inverted conversion, because the converted signal experiences reduced gain. The opposite occurs for inverted $\mathrm{XPolM}$, in which the signal inversion is enhanced by XGM. Figure 3(b) shows the results obtained from the counter-propagation setup. The penalty introduced to the inverted XPolM and XGM is reduced to approximately $0.5 \mathrm{~dB}$. This penalty is negligible if the insertion loss of the PBS in the counter-propagation setup is taken into consideration. However, the most significant variation between the co- and counter-propagation setups is in terms of the non-inverted XPolM. There is a reduction of approximately $4.5 \mathrm{~dB}$ in the power penalty for this technique between the two experimental setups. This result indicates that the NPR effect is present with less power in the counter-propagation configuration. This improvement in penalty was not found for inverted XPolM. This may be due to the PBS, which has an extinction ratio limited to $20 \mathrm{~dB}$. If the conversion has reached this limit then any improvement in the conversion, due to an increase in the NPR, will be undetected. The results presented in figure 5 are in contrast to those presented in [13], where the smallest power penalty was found to be in the non-inverted setup. This discrepancy may be 
due to the different relationships between the gain and polarization nonlinearities in the two devices. It should be noted that whilst the results presented in figure 5 are in contrast to [13], they are in agreement with the results presented in [14].
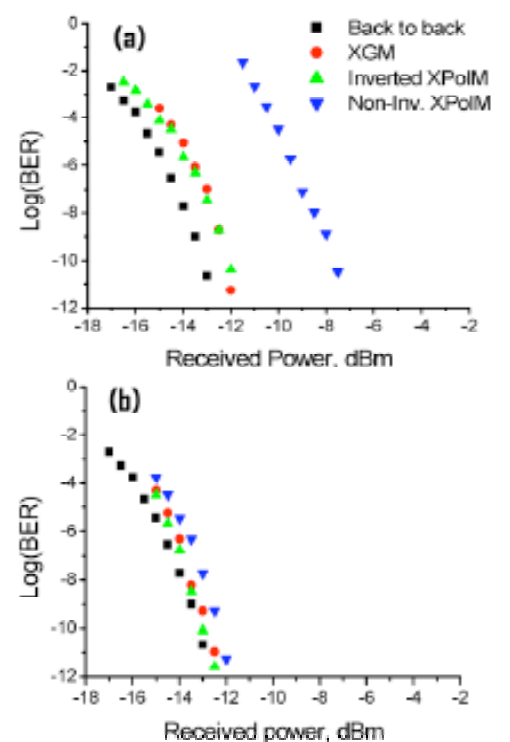

Figure 3. BER as a function of received converted power for XGM, inverted XPolM and non-inverted XPolM in (a) co- and (b) counter-propagation. Pump wavelength $=1538 \mathrm{~nm}$, probe wavelength $=1535 \mathrm{~nm}$.

The eye diagrams measured for the counter-propagation setup are shown in figure 4, along with the back-to-back measurement. The Q-factor measured for each setup is also shown. It can be seen that the Q-factor obtained for both inverted XPolM and XGM is practically the same as for the back-to-back case. From figure 4 it can be seen that a slow rising edge and a fast falling edge is present in the back-to-back eye diagram. It is logical to assume that the eye diagrams for the inverted configurations of XGM and inverted XPolM should therefore have a fast rising edge and a slow falling edge. Likewise, it is logical to assume the non-inverted XPolM configuration should have a slow rising edge and a fast falling edge, like the back-to-back eye diagram. However, from observation of figure 4 it appears that the opposite is true. This is caused by the presence of an inverted electrical amplifier used in the receiver. The pump power was also varied in both setups in order to optimize its value. The highest available pump power of $7 \mathrm{dBm}$ consistently led to the best XPolM performance. This is expected, once the device has not reached its NPR saturation point.
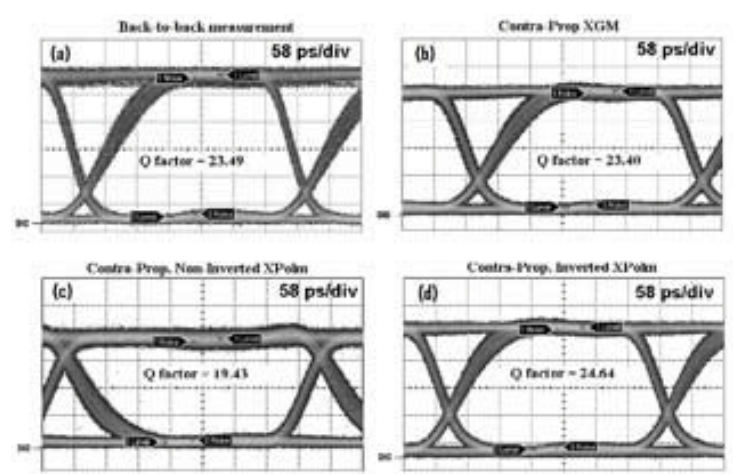

Figure 4. Eye diagrams for back-to-back, XGM, noninverted XPolM and inverted XPolM in counter-propagation.

\section{WAVELENGTH DEPENDENCE OF WAVELENGTH CONVERSION}

The BER as a function of received power for various probe signal wavelengths is shown in figure 5. From this figure it can be seen that the effect of varying the probe wavelength is strongly dependent on the system configuration. Wavelength independent conversion is found for non-inverted XPolM in the co-propagation setup, for BERs as low as $10^{-12}$, as can be seen from Fig. 5(a). For BERs lower than this a small wavelength dependence is introduced. The cause of the insensitivity to wavelength may be explained from the relationship between XGM and XPolM. In the non-inverted co-propagation setup the NPR acts to increase the extinction ratio of the converted signal. However, the suppressed gain causes a reduction of the extinction ratio, as it results in a lower amplification of the "1" level. As the probe wavelength is moved away from the gain peak of the SOA there are two effects on the wavelength conversion. Firstly, the NPR is reduced due to its intensity dependence. Secondly, the gain suppression is reduced. The result is that there is a balance in the contribution from these effects in the case of non-inverted XPolM in the co-propagation setup. Non-inverted XPolM is also obtained for the counterpropagation setup and is shown in figure 5(b). It has already been established that the NPR effect is larger for counter-propagation, which results in a wavelength dependence of the BER as the NPR and gain suppression mechanisms are no longer balanced. The increase in NPR in the counter-propagation setup results in a much larger contribution of NPR than of XGM. This leads to a wavelength dependence of $2.5 \mathrm{~dB}$ over the $35 \mathrm{~nm}$ span. Further comparison between figure 5(a) and figure 5(b) reveals that the wavelength insensitivity in co-propagation is at the expense of a larger power penalty. 

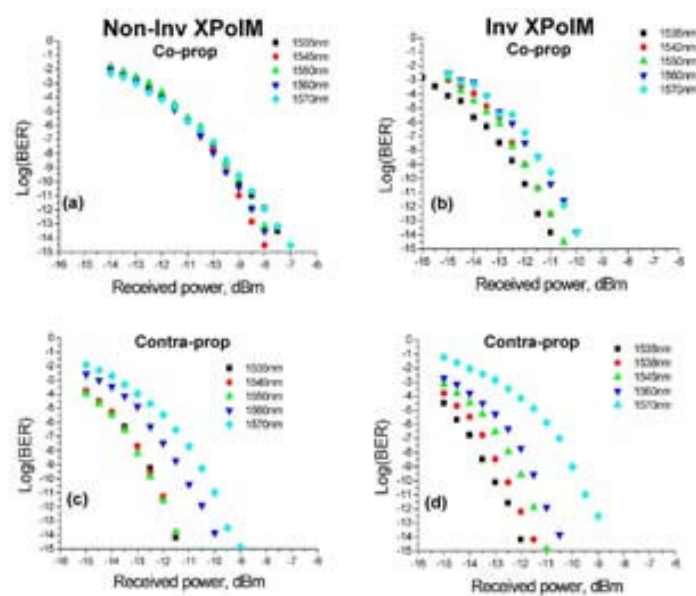

Figure 5. BER as a function of received power for various probe signals wavelengths, where (a)-(b) represent non-inverted XPolM and (c)-(d) represent inverted XPolM in co and counter-propagation, respectively.

The wavelength dependence of inverted XPolM is also analyzed. Obviously, wavelength insensitivity is not possible for inverted XPolM, as the NPR and gain suppression both increase the extinction ratio of the converted signal. In the co-propagation setup, as the wavelength is increased the power penalty increases by $1 \mathrm{~dB}$, as can be seen in figure 5(c). Similarly, in counter-propagation, the power penalty over the same wavelength range increases by approximately $3.5 \mathrm{~dB}$ as shown in figure 5(d). For both inverted and non-inverted conversion it can be seen that the wavelength dependence of the XPolM is smallest in co-propagation. In both cases the cause of the larger wavelength dependence in counter-propagation is the increase in the contribution of the wavelength dependent NPR effect. The insensitivity to wavelength is at the cost of a penalty of over $5 \mathrm{~dB}$ whereas inverted conversion in the counter-propagation setup has a minimum penalty of $0.26 \mathrm{~dB}$. The same trends are observed in the analysis of the Q-factor as a function of probe wavelength. The Q-factor in the co-propagation setup, for non-inverted XPolM, and for a received power of $10^{-9}$ was measured as 6.4 at $1535 \mathrm{~nm}$. The value at 1570 $\mathrm{nm}$ was measured to be 6.2 , a difference of only 0.2 in the Q-factor over a range of $35 \mathrm{~nm}$. In the non-inverted counter-propagation setup for the same experimental conditions a wavelength dependence in the Q-factor of 3.3 was measured over the same wavelength range. In each setup the highest Q-factor was measured for a probe wavelength of $1535 \mathrm{~nm}$.

\section{CONCLUSIONS}

The effect of NPR in bulk SOAs has been introduced in this paper. The main contributing factors have been discussed. This effect has been used to perform wavelength conversion based on XPolM. A comparison is made between co- and counter-propagation setups, in order to establish advantages and disadvantages of each. Both inverted and non-inverted XPolM were performed. The results indicate that the XPolM effect is larger in counterpropagation, with a $4.5 \mathrm{~dB}$ improvement in the penalty introduced for non-inverted conversion. The wavelength dependence of the conversion is determined over a span of $35 \mathrm{~nm}$. It is shown that wavelength insensitive conversion is possible for non-inverted XPolM in the copropagation setup over the accessible wavelength range, although a larger penalty is introduced in this case. The cause of this wavelength insensitivity is attributed to a balancing between NPR and gain suppression in the non-inverted case. It may be concluded that the question of whether to use a co- or counter-propagation setup for XPolM is dependent on the exact requirements of the system. If wavelength insensitivity is desired then co-propagation is more suitable, whereas if the power penalty is a crucial parameter then counter-propagation should be used.

\section{REFERENCES}

[1] E. de Argaez. "Internet world stats". August 2007. URLs: http://www.internetworldstats.com

[2] S. Tariq, M.K. Dhodhi, J.C. Palais and R.E. Ahmed. "Next generation DWDM networks: Demands, capabilities and limitations". IEEE Canadian Conf. on Elec. and Comp. Eng. 2000. Vol. 2, pp. 1003-1007. 2000.

[3] G. Held. "On the road to OC-768". IT Professional. Vol. 3, pp. 46-48. 2001.

[4] D. Cotter, R.J. Manning, K.J. Blow, A.D. Ellis, A.E. Kelly, D. Nesset, I.D. Phillips, A.J. Poustie and D.C. Rogers. "Nonlinear optics for high-speed digital information processing". Science. Vol. 286, pp. 1523-1528. 1999.

[5] D.M. Patrick, A.D. Ellis, D.A.O. Davies, M.C. Tatham and G. Sherlock. "Demultiplexing using polarization rotation in a semiconductor laser amplifier". IEE Electronic Letters. Vol. 30, pp. 341-342. 1994. 
[6] H. Soto, D. Erasme and G. Guekos. "Cross polarization modulation in semiconductor optical amplifiers". IEEE Photonic Tech. Letters. Vol. 9, pp. 449-451. 1997.

[7] R.J. Manning, A. Antonopoulos, R. Le Roux and A.E. Kelly. "Experimental measurement of nonlinear polarization rotation in semiconductor optical amplifiers". IEE Electronic Letters. Vol. 37, pp. 229-231. 2001.

[8] G.P. Agrawal, N.A. Olsson. "Self-phase modulation and spectral broadening of optical pulses in semiconductor laser amplifiers". IEEE J. Quantum Electronics. Vol. 25, pp. 2297-2306. 1989.

[9] C.H. Henry. "Theory of the linewidth of semiconductor lasers". IEEE J. Quantum Electronics. Vol. 18, pp. 259-264. 1982.

[10] P. Dousiere, P. Garabedian, C. Graver, D. Bonnevie, T. Fillion, E. Derouin, M. Monnot, J.G. Provost, D. Leclerc and M. Klenk. " $1.55 \mu \mathrm{m}$ polarization independent semiconductor optical amplifier with $25 \mathrm{~dB}$ fiber to fiber gain". IEEE Photonic Tech. Letters. Vol. 6, pp. 170-172. 1994.
[11] M. Itoh, Y. Shibata, T. Kakitsuka, Y. Kadota, and Y. Tohmori. "Polarization-insensitive SOA with a strained bulk active layer for network device application”. IEEE Photonic Tech. Letters. Vol. 14, pp. 765-767. 2002.

[12] C. Vassallo. "Polarization-independent antireflection coatings for semiconductor optical amplifiers". IEE Electronic Letters. Vol. 24, pp. 61-62. 1988.

[13] Y. Liu, M.T. Hill, E. Tangdiongga, H. de Waardt, N. Calabretta, G.D. Khoe and H.J.S. Dorren. "Wavelength conversion using nonlinear polarization rotation in a single semiconductor optical amplifier". IEEE Photonic Tech. Letters. Vol. 15, pp. 90-92. 2003.

[14] L. Guo and M.J. Connelly. "Signal induced birefringence and dichroism in a tensile-strained bulk semiconductor optical amplifier and its application to wavelength conversion". IEEE J. Light. Technology. Vol. 23, pp. 4037-4045. December 2005. 